\title{
Novo RMLSA com Tonificação de Circuito e ciente da Qualidade de Transmissão com Baixa Margem em Redes Ópticas Elásticas
}

\author{
Lucas R. Costa, Ítalo Barbosa Brasileiro e André C. Drummond \\ Departamento de Ciência da Computação - Universidade de Brasília (UnB), \\ 70910-900, Brasília, Brasil
}

\begin{abstract}
This paper evaluates the Quality of Transmission (QoT) margins adopted in traditional Routing, Modulation Level, and Spectrum Assignment (RMLSA) algorithms that quantify QoT through the maximum transparent range of modulation. An RMLSA algorithm is proposed that categorizes the minimum margins that must be adopted for the establishment of new circuits, based on their expected degeneration and the impact it will have on the already established optical circuits. Simulation results show that the proposed minimum margins associated with circuit invigoration present better results than algorithms that propose a fairer margin or evaluate QoT through the maximum transparent range.
\end{abstract}

Resumo. Este artigo avalia as margens de QoT (Quality of Transmission) adotadas em algoritmos RMLSA (Routing, Modulation Level, and Spectrum Assignment) tradicionais que quantificam o QoT por meio do alcance máximo transparente da modulação. É proposto um algoritmo RMLSA que categoriza as margens mínimas que devem ser adotadas para o estabelecimento de novos circuitos, tendo como base a sua degeneração esperada e o impacto causado aos circuitos ópticos já estabelecidos. Resultados de simulação mostram que as margens mínimas propostas associada a tonificação dos circuitos apresentam resultados melhores que algoritmos que propõem uma margem mais justa ou que avaliam o QoT por meio do alcance máximo transparente.

\section{Introdução}

Nos últimos anos, novos tipos de serviço e aplicações de rede, como computação em nuvem e streaming de alta definição, mantiveram o tráfego da Internet em constante crescimento. Atualmente, esforços tem sido aplicados para o desenvolvimento de tecnologias que permitam obter maior capacidade de transmissão e maior aproveitamento dos recursos nas grandes redes de transporte.

A tecnologia de Redes Ópticas Elásticas (Elastic Optical Networks - EON) surge como uma solução promissora para apoiar a crescente demanda de tráfego nas redes de alta velocidade. As principais características da EON é proporcionar uma maior flexibilidade e escalabilidade na alocação do espectro para os diversos serviços emergentes da Internet [Chatterjee et al. 2015]. As EONs são baseadas na multiplexação óptica por divisão de frequência ortogonal (Optical-Orthogonal Frequency Division Multiplexing O-OFDM). Esta tecnologia permite a divisão do espectro óptico em pequenos intervalos de frequência, denominados slots. Neste contexto, uma transmissão óptica é formada por 
uma quantidade arbitrária de slots no espectro, que são agrupados para a formação do canal no qual é estabelecido o que chamamos de circuito óptico [Chatterjee et al. 2015].

Em uma transmissão óptica, a margem de um circuito óptico pode ser quantificada como a diferença entre a qualidade da transmissão (Quality of Transmission - QoT) do sinal e o limite ao qual o sinal é considerado recuperável. Às imperfeições da camada física provocam uma degradação do sinal óptico à medida que ele se propaga na rede. A acumulação destas degradações, ao longo da rota do circuito óptico, pode tornar a taxa de erro de bit (Bit Error Rate - BER) intolerável quando o sinal chega ao receptor, impossibilitando a transmissão óptica [Beyranvand and Salehi 2013]. Em EON, a literatura usualmente afere a QoT por meio da relação sinal ruído (Signal-to-Noise Ratio SNR) ou pelo alcance máximo transparente estimado para a modulação adotada no circuito [Pointurier 2017].

Modelos que medem a QoT por meio do alcance máximo transparente da modulação podem não garantir um desempenho adequado, pois ainda assim podem ocorrer níveis altos de BER, o que torna inviável a comunicação em um cenário real. Por outro lado, tais modelos também podem superdimensionar a margem de QoT, estabelecendo circuitos com valores de BER muito baixos, subutilizando a capacidade da rede óptica. Nesse contexto, modelos que levam em consideração as interferências da camada física tornamse mais adequados para aferição da QoT do circuito óptico, pois levam em consideração o alcance transparente do sinal, a interferência do meio de propagação e dos circuitos já estabelecidos, bem como a degradação do canal ao logo da rota. Explorar eficientemente estes fatores contribui para a elaboração de técnicas mais eficientes no desenvolvimento de soluções de engenharia de tráfego das redes de núcleo.

Este artigo faz uma avaliação das margens de QoT utilizadas em circuitos ópticos EON com base em um modelo analítico para o cálculo de SNR. São categorizadas as margens mínimas que devem ser adotadas para o estabelecimento de novos circuitos, tendo como base a sua degeneração esperada e o impacto que ele causará aos circuitos ópticos já estabelecidos. Com base nesta categorização, este artigo propõe um novo algoritmo de alocação de espectro, chamado Low-Margin with Circuit Invigorating - LMCI, que tonifica o circuito óptico por meio da utilização de níveis de modulação mais robustos, sem comprometimento do uso de espectro, associando a escolha da modulação a uma baixa margem de QoT. Os resultados de simulação mostram que o algoritmo proposto apresenta resultados melhores que algoritmos da literatura que propõem uma margem mais justa ou que avaliam o QoT por meio do alcance máximo transparente. A proposta mostrou uma redução em média de $33 \%$ em termos de bloqueio de chamadas e um desempenho de complexidade de tempo semelhante a algoritmos que consideram o alcance máximo na escolha da modulação.

As principais contribuições deste artigo são: (i) avaliar as margens de QoT adotadas em algoritmos tradicionais que levam em consideração a escolha da modulação por meio da distância, mostrando que em determinados cenários de alcance transparente as margens são superdimensionadas; (ii) são quantificadas as margens mínimas que devem ser adotadas no estabelecimento de novos circuitos ópticos da rede; e (iii) é apresentado um novo algoritmo de alocação de espectro com tonificação de circuitos e QoT com baixa margem. 


\section{Redes Ópticas Elásticas}

As redes ópticas elásticas emergem como solução para o problema do crescimento de requisitos de banda. A transmissão do sinal óptico em diferentes intervalos de frequência viabiliza a formação de múltiplos circuitos em paralelo e independentes [Chen et al. 2015]. Além disso, as EON também se beneficiam da flexibilidade espectral, na qual a divisão do espectro óptico em slots de baixa granularidade $(12,5 \mathrm{GHz})$ permite maior ajuste do canal óptico à banda requisitada para cada circuito [Chen et al. 2015].

O estabelecimento de circuitos em uma EON requer a reserva de recursos espectrais. A escolha dos recursos que serão aplicados ao novo circuito constitui o problema de roteamento, escolha de modulação e de espectro (Routing, Modulation level and spectrum assignment - RMLSA) [Chen et al. 2015]. Para solucionar o problema RMLSA, o circuito passa por uma avaliação, feita pelo algoritmo RMLSA, na qual serão definidos os recursos adequados para a formação do novo canal óptico. Na literatura relacionada a EON, o problema RMLSA é um tema clássico, e existem várias soluções RMLSA propostas, que consideram diferentes características da rede por meio de diversas abordagens. A Figura 1 apresenta uma demonstração do problema RMLSA em uma topologia simples.

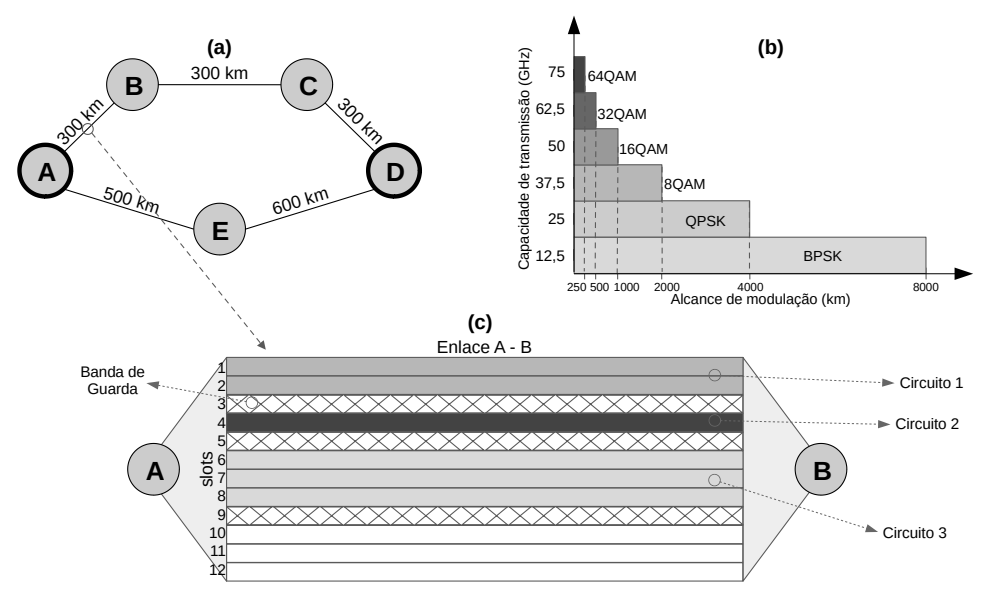

Figura 1. Problema de (a) roteamento, (b) escolha de modulação e (c) alocação espectral.

O problema RLMSA pode ser dividido em três partes, que devem ser solucionadas na sequência descrita a seguir. A primeira parte é o problema de roteamento (Figura 1 (a)), em que é escolhida uma rota para o circuito, por meio de alguma heurística, como menor número de saltos ou menor distância. Na Figura 1 (a), é utilizado como exemplo o par de nós $A-D$. A rota de menor caminho é composta pelos nós $A-B-C-D$, e a rota de menor salto é composta pelos nós $A-E-D$. A segunda parte do problema é a escolha do formato de modulação que será aplicado ao circuito. Normalmente a literatura opera a escolha do formato de modulação por meio do alcance máximo transparente pré-definido [Chatterjee et al. 2015, Chen et al. 2015]. Algoritmos que utilizam esse estratégia escolhem a modulação de maior eficiência espectral que tenha limiar de distância maior ou igual ao comprimento da rota. No caso ilustrado na Figura 1 (b), a modulação mais eficiente para a rota $A-B-C-D$ é a 16QAM, enquanto para a rota $A-E-D$, a modulação mais eficiente é a 8QAM. Uma vez definida qual modulação será utilizada, pode-se obter a quantidade de slots necessários para atender uma dada demanda em $\mathrm{Gb} / \mathrm{s}$. Com isso a terceira parte do problema é a escolha da frequência óptica para a alocação, 
em outras palavras quais slots serão utilizados no circuito. A escolha de slots deve seguir alguma política de alocação, como First Fit, Last Fit, Best Fit ou outras. No exemplo da Figura 1 (c), uma chamada de requisição de circuito com $100 \mathrm{~Gb} / \mathrm{s}$ necessita de 3 slots, caso utilize a modulação 8QAM, e 2 slots, se a modulação aplicada for 16QAM. Para obter uma maior eficiência espectral, os trabalhos que utilizam modulação adaptativa sempre optam pela utilização da modulação mais eficiente, já que menos slots são utilizados nesses casos. É importante destacar a utilização de bandas de guarda entre os circuitos alocados (ilustradas nos slots 3, 5 e 9 da Figura 1 (c)), para reduzir a interferência entre circuitos adjacentes.

Para reduzir a utilização de transmissores e receptores da rede, e também reduzir o atraso inserido por conversões óptico-elétrico-ópticas (OEO), é preciso considerar as restrições ópticas na fase de alocação do circuito. As restrições consideradas em uma rede EON transparente são duas [Chatterjee et al. 2015]: a restrição de continuidade exige que o circuito permaneça por toda a rota nos mesmos índices de slots alocados, evitando a ocorrência de conversão OEO para a alteração da frequência utilizada pelo circuito; e a restrição de contiguidade, que exige que os slots alocados para o circuito sejam adjacentes no espectro, evitando assim a utilização de múltiplos transmissores e receptores para um mesmo circuito em diferentes canais dispersos no espectro óptico. Além da definição de um alcance máximo transparente da modulação, para medir a QoT de um circuito, alguns trabalhos utilizam um modelo para representar e medir as imperfeições e interferências da camada física na potência do sinal óptico. A modelagem permite ter uma maior proximidade entre o cenário de simulação e um modelo real de EON. A seção a seguir apresenta a modelagem de camada física utilizada neste trabalho e adotada nos algoritmos avaliados.

\subsection{Modelo de Camada Física}

A adoção de um modelo que represente as imperfeições da camada física leva para mais próximo da realidade o cenário estudado. Assim, é possível propor soluções e apresentar medições que tenham mais chances de permanecerem válidas e, ao mesmo tempo, eficientes ao serem aplicadas em uma EON real. Os autores em [Johannisson and Agrell 2014] propõem uma modelagem para o cálculo da qualidade do sinal de um circuito a partir da relação entre sinal e ruído (Signal to noise ratio - SNR). O SNR de um circuito $i$ pode ser medido de acordo com a equação:

$$
S N R_{i}=\frac{G}{G_{A S E}+G_{N L I}}
$$

onde $G$ é a densidade espectral da potência do sinal (PSD - Power Spectral Density), definido por $G=P_{t x} / \Delta_{f}$, em que $P_{t x}$ é a potência de sinal e $\Delta_{f}$ é a largura de banda do circuito. A PSD do ruído ASE (Amplified Spontaneous Emission) $\left(G_{A S E}\right)$ é definida pela equação:

$$
G_{A S E}=\sum_{l \in r_{i}} N_{l}\left(G_{A M P}-1\right) F h v
$$

em que $N_{l}$ representa o número de spans do enlace $l$ pertencente à rota $r$ utilizada pelo circuito $i, G_{A M P}$ representa o ganho do amplificador óptico, $F$ é o fator de emissão espontânea, $h$ é a constante de Planck e $v$ é a frequência da luz. Por sua vez, a PSD dos 
ruídos não-lineares (NLI) pode ser calculada por:

$$
G_{N L I}=\sum_{l \in r_{i}} N_{l} G_{N L I}^{l}
$$

em que $G_{N L I}^{l}$ é a densidade espectral da potência de um span do enlace $l$, calculada por:

$$
G_{N L I}^{l}=\frac{3 \gamma^{2} I^{3}}{2 \pi \alpha\left|\beta_{2}\right|}\left(\operatorname{arcsinh}\left(\frac{\pi^{2}\left|\beta_{2}\right| B_{i}^{2}}{2 \alpha}\right)+\sum_{j} \ln \left[\frac{\Delta f_{i j}+\frac{B_{j}}{2}}{\Delta f_{i j}-\frac{B_{j}}{2}}\right]\right)
$$

onde $j$ é o circuito vizinho no link $l, B_{i}$ é a largura de banda do circuito $i$ em questão, $B_{j}$ é a largura de banda do circuito $j$ em questão, $\Delta_{f i}$ é o espaçamento da frequência central entre $i$ e $j, \gamma$ é o coeficiente de não-linearidade da fibra, $\beta_{2}$ é o parâmetro de dispersão da fibra e $\alpha$ é a atenuação de potência da fibra.

Ao considerar o cálculo de SNR para medir a qualidade do circuito, a literatura também atribui limiares de SNR para os diferentes formatos de modulação. Assim, em simulações que consideram as interferências de camada física e a verificação de SNR do circuito pelo cálculo apresentado na Eq. 1, a escolha de modulação por meio do limiar de distância na técnica de modulação adaptativa é substituida pela escolha baseada no limiar de SNR. Com essa alteração, para um circuito apresentar qualidade de transmissão aceitável, ele precisa apresentar valor de SNR acima do limiar da modulação adotada. Os limiares de SNR utilizados são apresentados na Tabela 1 [Fontinele et al. 2017], juntamente com a respectiva capacidade de transmissão e os limiares de distância [Costa and Drummond 2017].

Tabela 1. Limiares de SNR e de distância adotados na literatura. Capacidade de Limiar de distância Limiar de SNR

\begin{tabular}{r|ccc}
\multicolumn{2}{c}{ Transmissão/slot $(\mathrm{Gb} / \mathrm{s})$} & $(\mathrm{km})$ & $(\mathrm{dB})$ \\
\hline BPSK & 12,5 & 8000 & 6 \\
\hline QPSK & 25,0 & 4000 & 9 \\
\hline 8QAM & 37,5 & 2000 & 12 \\
\hline 16QAM & 50,0 & 1000 & 15 \\
\hline 32QAM & 62,5 & 500 & 18 \\
\hline 64QAM & 75,0 & 250 & 21 \\
\hline
\end{tabular}

Além de atender o limiar de SNR do formato de modulação adotado, o novo circuito a ser estabelecido na rede deve também manter acima do limiar o SNR dos circuitos com os quais compartilha enlaces. Para isso, o SNR deve ser recalculado para os circuitos que já estão ativos na rede, quando há a tentativa de estabelecimento de um novo circuito em seus enlaces. Se o novo circuito levar para abaixo do limiar o SNR de algum circuito já estabelecido, então o novo circuito não poderá ser estabelecido. Neste caso, devem ser explorados novos recursos (faixa de slots, rota ou modulação) para o novo circuito, ou ele não poderá ser estabelecido, provocando o bloqueio da demanda requisitada.

\section{Trabalhos Relacionados}

Recentemente, há um número crescente de trabalhos de pesquisa que investigam soluções para o problema RMLSA em EON transparentes. O problema vem sendo tratado 
de diversas formas sob diversos cenários de rede, incluindo ainda considerações sobre a tecnologia de modulação e diferentes limiares adaptativos no canal.

O problema da escolha de modulação foi inicialmente apresentado em [Christodoulopoulos et al. 2011], no qual os autores adicionam uma variável a mais no problema de roteamento e de atribuição do espectro (Routing and Spectrum Assignment - RSA) das EONs. Posteriormente, o problema RMLSA também foi investigado em [Wan et al. 2012], no qual os autores avaliam os efeitos da modulação adaptativa em algoritmos RSA. Em ambos os trabalhos, é considerado o alcance máximo transparente da modulação como meio de medir a QoT do circuito a ser estabelecido. Neste contexto, após a definição de uma rota, o algoritmo atribui como modulação mais adequada aquela que tenha limiar de distância (pré-estabelecido) maior ou igual ao comprimento da rota.

Os autores em [Fontinele et al. 2017] propõem um novo algoritmo RMLSA que considera os efeitos da camada física para EON transparente, chamado K-Shortest Path with Reduction of QoTO (KSP-RQoTO). O algoritmo busca reduzir a degradação de QoT dos circuitos já estabelecidos na rede por meio da adoção de uma margem fixa no estabelecimento de novos circuitos. Além disso, o KSP-RQoTO procura reduzir essa interferência mediante a alocação de rotas que degradam menos os circuitos ópticos que já estão ativos na rede e são mais frágeis em relação ao SNR. Para isso, o algoritmo utiliza como escolha de rota os k-menores caminhos (k-shortest path) para o par de nós origem e destino da requisição. Diversos algoritmos são comparados em diferentes cenários de rede. Os resultados de simulação mostram que a proposta apresenta melhor desempenho que os algoritmos comparados em relação a probabilidade de bloqueio e justiça do serviço.

Os mesmos autores em [Fontinele et al. 2018] propõem outro algoritmo RMLSA, chamado Spectrum Assignment with Interference Reduction (SAIR), que considera o mesmo modelo de representação de imperfeições da camada física. O objetivo do SAIR é alocar faixas de espectro que causem menos interferências na QoT dos circuitos já estabelecidos na rede. Os autores realizam um estudo avaliando o desempenho do algoritmo proposto com diversos algoritmos de alocação de espectro da literatura. Contudo, todos os algoritmos comparados utilizam como escolha da rota o menor caminho (shortest path) para o par de nós origem e destino da requisição. Para escolha da modulação, os algoritmos avaliados utilizam o nível de modulação mais eficiente espectralmente que alcança um QoT aceitável na rede. Não é utilizada nenhuma margem de segurança para evitar a degradação de QoT dos circuitos já estabelecidos. Os resultados de simulação mostram que o SAIR, como algoritmo de alocação de espectro, obtém desempenho superior aos demais algoritmos de alocação em termos de probabilidade de bloqueio de circuito e probabilidade de bloqueio de banda.

Em [Brasileiro et al. 2019], os autores propõem um algoritmo de alocação de regeneradores que adapta o formato de modulação do circuito fortalecendo e reduzindo a interferência caudada pelos futuros circuitos que serão estabelecidos na rede. A proposta visa utilizar um formato de modulação mais robusto sem comprometer o uso do espectro. Dessa forma, modulações mais robustas com uma margem de QoT maior podem ser utilizadas desde que seu uso não comprometa a quantidade de slots utilizadas pelo circuito. Os resultados de simulação comprovaram que o algoritmo proposto obteve melhor desempenho que algoritmos da literatura que alocam regeneradores na rede. 
Tendo em vista o estado da arte apresentado, este trabalho propõe um novo algoritmo de alocação de espectro que procura reduzir a degradação de QoT dos circuitos já estabelecidos na rede por meio da adoção de uma margem relativa à modulação no momento do estabelecimento do circuito. Associado a esta margem de QoT proposta, o algoritmo ainda propõe o fortalecimento do circuito óptico por meio da utilização de níveis de modulação mais robustos.

\section{QoT com Baixa Margem em EON}

Para compreender os limites mínimos de margem que podem ser adotadas na criação de novos circuitos ópticos EON, este trabalho faz uma avaliação das margens de QoT usualmente utilizadas por algoritmos da literatura que medem o QoT de circuito por meio do alcance máximo transparente da modulação. A Seção a seguir apresenta esta avaliação em diversos cenários de alcance máximo transparente para as modulações mais utilizadas na literatura. Em seguida, é apresentado o algoritmo proposto neste trabalho tendo como base as margens mínimas de QoT para a alocação de novos circuitos EON.

\subsection{Margens de QoT Usualmente Utilizadas}

Os cenários considerados de alcance máximo transparente realizados nos testes foram cenários normalmente utilizados pela literatura em artigos científicos que estudam o problema RMLSA. Alguns cenários foram retirados dos artigos [Guo et al. 2015, Feng et al. 2017, Walkowiak et al. 2017]. Os cenários de 5 a 7 foram avaliados apenas com a finalidade de observar os limites de distância nos quais a QoT não é atendida para até $100 \%$ dos circuitos ópticos. A Tabela 2 apresenta os cenários considerados na avaliação com alcance máximo transparente em $\mathrm{km}$.

Tabela 2. Cenários considerados com alcance máximo transparente em km

\begin{tabular}{|l|c|c|c|c|c|c|}
\cline { 2 - 7 } \multicolumn{1}{c|}{} & BPSK & QPSK & 8QAM & 16QAM & 32QAM & 64QAM \\
\hline Cenário(1) & 4000 & 2000 & 1000 & 500 & 250 & 125 \\
\hline Cenário(2) & 8000 & 4000 & 2000 & 1000 & 500 & 250 \\
\hline Cenário(3) & 9000 & 4500 & 2250 & 1125 & 562 & 281 \\
\hline Cenário(4) & 9600 & 4800 & 2400 & 1200 & 600 & 300 \\
\hline Cenário(5) & 9800 & 4900 & 2450 & 1225 & 612 & 306 \\
\hline Cenário(6) & 10000 & 5000 & 2500 & 1250 & 625 & 312 \\
\hline Cenário(7) & 12000 & 6000 & 3000 & 1500 & 750 & 375 \\
\hline
\end{tabular}

Para as simulações realizadas nesta avaliação e na Seção 5, foi utilizado o simulador ONS [Costa and Drummond 2020]. O algoritmo RMLSA considerado para avaliação foi o FF-Dis [Wan et al. 2012]. Este algoritmo escolhe a rota por meio dos k-menores caminhos, em que $k=3$. A escolha do formato de modulação é a mais eficiente que atenda o seu alcance máximo pré-estabelecido no cenário. A politica de alocação de espectro considerada foi o First Fit (FF) por ser mais simples e comum. Cada simulação foi realizada cinco vezes utilizando o método de replicações independentes. Para os resultados apresentados foram calculados intervalos de confiança com $95 \%$ de confiabilidade. Em cada simulação foram geradas $10^{5}$ requisições de conexão com 6 níveis de granularidade: $25 \mathrm{~Gb} / \mathrm{s}, 50 \mathrm{~Gb} / \mathrm{s}, 100 \mathrm{~Gb} / \mathrm{s}, 200 \mathrm{~Gb} / \mathrm{s}, 300 \mathrm{~Gb} / \mathrm{s} \mathrm{e} 400 \mathrm{~Gb} / \mathrm{s}$ com as proporções 6:5:4:3:2:1, respectivamente. O processo de chegada das chamadas segue a distribuição de Poisson com origem e destino distribuídos uniformemente para todos os pares de comunicação.

As topologias consideradas nas simulações foram a USANet e a topologia PanEuro. A Figura 2 apresenta as distâncias dos enlaces em quilômetros. A largura de cada 
slot considerada foi $12,5 \mathrm{GHz}$ e foi assumido que cada enlace possui a capacidade de 320 slots. Assume-se uma banda de guarda de 2 slots. Cada nó na topologia é equipado com transmissores e receptores suficientes sendo cada transmissor capaz de transmitir até 32 slots (correspondente à transmissão da maior banda, em BPSK). As modulações consideradas são BPSK, QPSK, 8QAM, 16QAM, 32QAM e 64QAM com 1, 2, 3, 4, 5 e 6 bits por símbolo respectivamente. Para o cálculo de SNR, os seguintes parâmetros foram utilizados [Johannisson and Agrell 2014, Fontinele et al. 2017]: $N F=6 d B, h=$ $6,626 \times 10^{-34}, v=193 \times 10^{12}, G_{A M P}=\alpha \times L, L=100 \mathrm{~km}, \alpha=0,2 \mathrm{~dB} / \mathrm{km}, \gamma=$ $1,22(W \mathrm{~km})^{-1}, \beta_{2}=16 \times 10^{-24} \mathrm{ps}^{2} / \mathrm{km}^{\prime}$. Diversas cargas de trafego foram avaliadas e todas apresentaram resultados semelhantes. A avaliação apresentada se baseia em um único valor de carga, igual a 300 Erlangs.
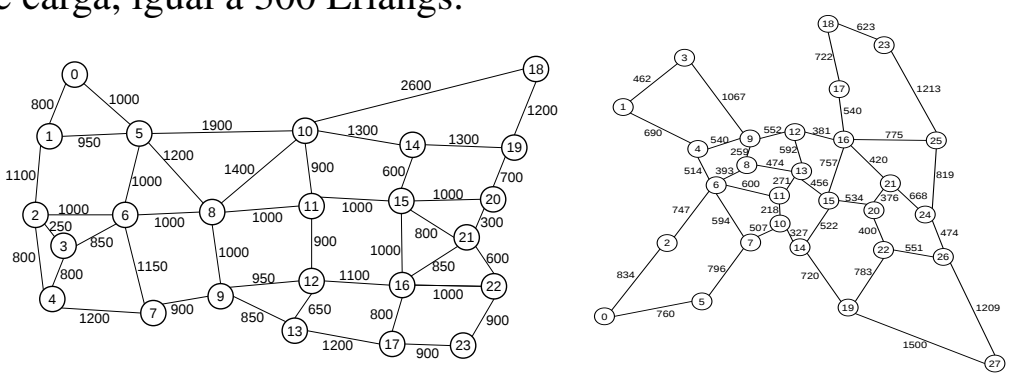

Figura 2. Topologias de rede consideradas, USA e PanEuro, respectivamente

Na Figura 3 (a), foi realizado o estudo da QoT dos circuitos em função da variação dos limiares de distância das modulações. Para medir a QoT, foi utilizada a Eq. 1. Entretanto, nesse cenário não foram considerados os bloqueios por baixa QoT, eles apenas foram contabilizados. Com essa simulação, é observado que entre os cenários (1) e (3) ocorrem poucos ou nenhum circuito sem QoT aceitável, e esses indicam que os limiares adotados nesses cenários podem ser adequados como substitutos para o cálculo de SNR.

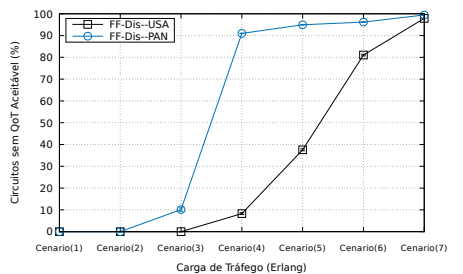

(a) Circuitos sem QoT Aceitável

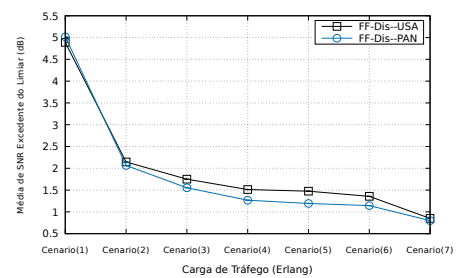

(b) SNR Excedente do Limiar

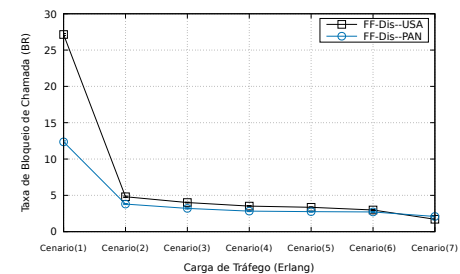

(c) Taxa de Bloqueio de Chamada

Figura 3. Avaliação de diferentes limiares de distância para os formatos de modulação.

A Figura 3 (b) apresenta a média do valor excedente (margem) de SNR dos circuitos estabelecidos, em relação ao limiar de SNR das modulações, considerando os diferentes cenários de distância da Tabela 2. Para o cenário (1), nota-se que há grande média de excedente (aproximadamente $5 d B$ ), o que indica que neste cenário, os circuitos que atendem os limiares de distância apresentam valor de SNR bem acima do limiar definido para as modulações, subdimensionando os recursos da rede. O baixo alcance aplicado às modulações também impede o estabelecimento de circuitos que seriam estabelecidos, caso o limiar de SNR fosse considerado ao invés do limiar de distância na verificação de QoT. A partir do cenário (2), para as duas topologias há pouca variação de excedente para os cenários avaliados. Em conjunto com os resultados da Figura 3 (a), os cenários (2) e (3) seguem como os mais próximos para substituir o cálculo de SNR. A Figura 3 (c) 
apresenta a taxa de bloqueio de chamadas para os cenários avaliados. No cenário (1) há maior taxa de bloqueio, por conta da aplicação de baixo alcance de distância para os circuitos. Com isso, circuitos que não atendem o limiar de distância ocorrem com maior frequência, resultando em maior bloqueio. A partir do cenário (2), há uma estabilização da taxa bloqueio.

Levando em consideração a avaliação realizada (Figura 3), os cenários (2) e (3) apresentam os alcances de distância mais indicados para a aferição de uma boa margem de QoT. A margem indicada para o estabelecimento de novos circuitos é próximo a $2 \mathrm{~dB}$ a mais que o limiar indicado na Tabela 1. Tendo como base esta avaliação, este trabalho considera a utilização de uma margem relativa na escolha do formato de modulação do algoritmo RMLSA. A margem é considerada relativa, pois escolhe-se uma valor percentual tendo como base a modulação considerada. Isto que dizer que modulações mais eficientes espectralmente, que por sua vez possuem limiares de SNR maiores devem adotar uma margem de segurança QoT maior devido sua sensibilidade de degradação. Em contrapartida, modulações mais robustas que possuem limiares de SNR menores, podem adotar margens de segurança QoT menores de forma que a margem média fique entre os limites apresentados nos cenários (2) e (3).

\subsection{Algoritmo proposto}

O algoritmo proposto neste trabalho tem como objetivo a criação de circuitos mais resistentes, que apresentam maior tolerância às interferências de camada física e ao ruído acrescentado pela criação de novos circuitos. A resistência é aumentada por meio da formação de circuitos que apresentam valor de SNR não tão próximos do limiar de SNR da modulação aplicada. Além disso, a escolha de modulação passa por um processo de tonificação, a fim de formar circuitos ainda mais resilientes. A Figura 4 apresenta o fluxograma do algoritmo Low-Margin With Circuit Invigorating (LMCI).

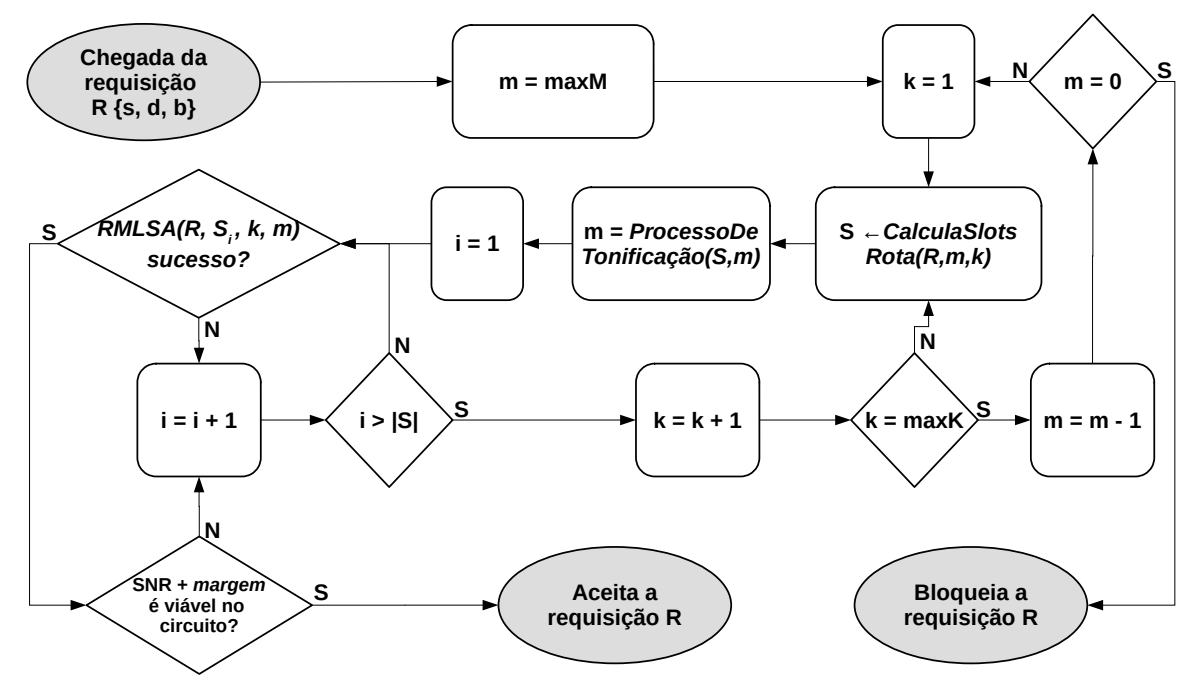

Figura 4. Fluxograma do algoritmo Low-Margin with Circuit Invigorating (LMCl).

O algoritmo LMCI considera a utilização de k-menores caminhos (KSP) para roteamento. Inicialmente, o algoritmo escolhe a modulação mais eficiente disponível na rede $(\max M)$. Em seguida, escolhe-se a primeira rota candidata (obtida pelo KSP) para ser percorrida. Para a rota e modulação atual, o conjunto de slots disponíveis da rota $(S)$ é calculado (CalculaSlotsRota $(R, m, k)$ ), e em seguida o formato de modulação passa 
pela tentativa de tonificação (ProcessoDeTonificação $(S, m)$ ). A tonificação de circuitos permite criar circuitos mais resistentes ao ruído causado pela formação de futuros novos circuitos [Brasileiro et al. 2019]. A tonificação ocorre pela alteração do formato de modulação para um formato mais robusto (menor valor de $m$ ), e que utilize a mesma quantidade de slots do formato atual. Com isso, a utilização espectral permanece a mesma, mas o limiar de SNR do circuito é alterado para um mais resistente. Após a tentativa de fortalecimento do circuito, é iniciada a avaliação dos slots alocáveis pelo circuito. Os slots alocáveis (conjunto $S$ ) são ordenados pela política First Fit, e para cada um é feita a verificação se é possível resolver o problema $\operatorname{RMLSA}\left(R M L S A\left(R, S_{i}, k, m\right)\right)$. Resolvendo o problema RMLSA, o valor adotado para o limiar de SNR do novo circuito é acrescentado com uma margem, que dá o nome ao algoritmo. $\mathrm{O}$ valor adicionado, referente a margem, corresponde a um percentual do limiar de SNR para a modulação avaliada ${ }^{1}$. Assim, o valor da margem se torna mais dinâmico, já que as modulações mais frágeis, que apresentam limiar de SNR mais alto, terão uma margem maior para o estabelecimento do circuito. $\mathrm{O}$ circuito é estabelecido se o seu valor de SNR adicionado à margem é viável e se os outros circuitos já estabelecidos na rede continuam a apresentar valor de SNR dentro do limiar. Destaca-se que nessa fase a margem não é adicionada na verificação do SNR dos circuitos já existentes na rede, pois estes já tiveram a margem considerada durante a seu estabelecimento e ela servirá como uma segurança para o impacto da formação dos novos circuitos. Se o SNR adicionado à margem não é adequado para o slot avaliado $\left(S_{i}\right)$, o próximo slot é escolhido, até que se esgotem os slots alocáveis da rota candidata sob avaliação. Neste ponto, o algoritmo ainda verifica se existem outras rotas candidatas para serem avaliadas. Se as rotas candidatas foram esgotadas para a modulação em avaliação, verifica se ainda existe modulação de menor eficiência espectral do que a modulação avaliada atualmente. Se ainda existem modulações disponíveis, então o algoritmo reinicia a avaliação, com a modulação um nível menor do que a modulação atual. Se ainda não existem formatos de modulação a avaliar, a chamada de circuito é bloqueada.

A complexidade de tempo do algoritmo LMCI é analisada como segue. O fato do algoritmo avaliar a possibilidade de criação de um novo circuito óptico para cada modulação $M$ em cada rota $K$ para todos os slots $S$ possíveis de um enlace, tem-se uma complexidade de pior caso sendo $O(M * K * S)$. A tonificação do circuito, compõe uma complexidade de tempo de $O(M)$, uma vez que é necessário avaliar todas as modulações na tonificação no pior caso. Para encontrar os slots alocáveis na rota, tem-se uma complexidade de tempo de $O(E * S)$, onde $E$ é quantidade de enlaces na rede. Ao compor todas as etapas, pode-se dizer que a complexidade de tempo do algoritmo LMCI é dada por $O\left(M^{2} * S^{2} * K * E\right)$.

\section{Avaliação de Desempenho}

As simulações foram realizadas com o objetivo de avaliar o desempenho do algoritmo LMCI proposto em comparação com três algoritmos da literatura, FFDis [Wan et al. 2012], RQoTO [Fontinele et al. 2017] e SAIR [Fontinele et al. 2018]. Para todos os algoritmos foram utilizados como escolha da rota o algoritmo clássico KSP de k-menores caminhos. Para os algoritmos FF-Dis, RQoTO e LMCI foram utilizados

\footnotetext{
${ }^{1} \mathrm{O}$ percentual escolhido depende fortemente da topologia de rede utilizada, de forma que é necessário uma avaliação experimental previa para definir o percentual adequado que corresponda as margens mínimas indicadas na Figura 3 (b).
} 
a politica de alocação First Fit. Dos algoritmos avaliados, somente o FF-Dis avalia o QoT do novo circuito por meio do alcance máximo transparente da modulação os demais algoritmos avaliam o QoT por meio do modelo que calcula os limiares de SNR de [Johannisson and Agrell 2014].

\subsection{Avaliação preliminar}

Para os algoritmos RQoTO, SAIR e LMCI, são necessários avaliações preliminares para definir parâmetros internos destes algoritmos. Os parâmetros internos do RQoTO referem-se a margem fixa que pode ser utilizada em alguns casos no algoritmo, representado por $\sigma$. Esta margem fixa representa um valor em $\mathrm{dB}$ acima do limiar de SNR para a escolha do circuito candidato que será estabelecido na rede. Os parâmetros internos do SAIR são referentes as taxas em $\mathrm{Gb} / \mathrm{s}$ utilizadas nas requisições de rede do cenário de simulação proposto. Assim, é escolhida uma determinada taxa para indicar se a politica de alocação para a demanda será First Fit ou Last Fit. Por exemplo, se a taxa escolhida for $200 \mathrm{~Gb} / \mathrm{s}$, todas as demandas requisitadas com $200 \mathrm{~Gb} / \mathrm{s}$ ou mais adotaram a politica First Fit enquanto as demandas requisitadas com menos de $200 \mathrm{~Gb} / \mathrm{s}$ adotaram a politica Last Fit. Para o algoritmo LMCI, os parâmetros internos referem-se à margem relativa adotada para todos os circuitos estabelecidos na rede. Esta margem relativa representa um valor percentual em dB acima do limiar de SNR para a modulação do circuito candidato que será estabelecido na rede.

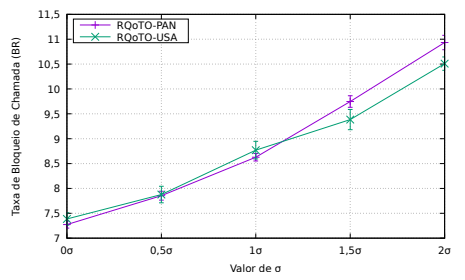

(a) RQoTO

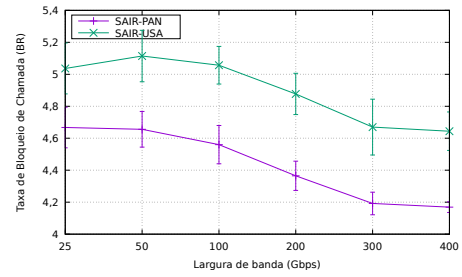

(b) SAIR

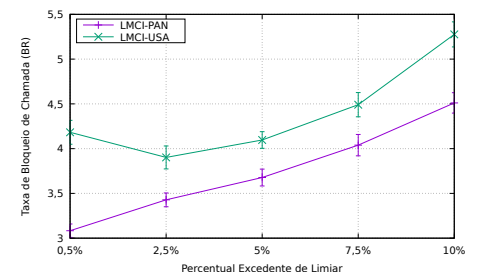

(c) LMCI

Figura 5. Avaliação dos parâmetros internos dos algoritmos avaliados.

A Figura 5 apresenta avaliação dos parâmetros internos dos algoritmos RQoTO, SAIR e LMCI, respectivamente para uma carga de 400 Erlangs no cenário de simulação descrito na Seção 4.1 para as topologias USA e PanEuro. Observa-se que para o algoritmo RQoTO, o valor de $\sigma$ que obteve o melhor desempenho no cenário proposto foi o de 0 . Isto significa que para ambas as topologias a margem fixa adotada no algoritmo prejudica seu desempenho. Portanto para a próximas simulações foram utilizados o valor de $\sigma=0$ para o RQoTO. Para o algoritmo SAIR, a taxa que obteve o melhor desempenho em ambas a topologias foi $300 \mathrm{~Gb} / \mathrm{s}$. Isso significa que as requisições de 300 e $400 \mathrm{~Gb} / \mathrm{s}$ utilizaram a politica de First Fit, a demais utilizaram Last Fit na composição do algoritmo. Para o algoritmo proposto LMCI, a margem relativa melhor avaliada foi de 2,5\% para a topologia USA e $0,5 \%$ para a topologia PanEuro. Dessa forma, todos os caminhos ópticos que serão estabelecidos devem ter uma margem mínima pelo menos acima do limiar deste percentual. O fato do valor ser diferente na topologia PanEuro refere-se a sua característica mais restrita com enlaces menores, o que possibilita o estabelecimento de circuitos com níveis de modulação mais eficientes quando a margem é baixa. Quando utiliza-se margens mais altas o algoritmo começa a utilizar níveis de modulação mais robustos que, por sua vez, utilizam mais espetro dificultando sua alocação. 


\subsection{Média de SNR Excedente do Limiar (dB)}

A média de SNR excedente do limiar (Figura 6) apresenta a margem mínima em média adotada nos circuitos estabelecidos na rede. Este valor refere-se a diferença entre o valor do SNR do circuito e seu limiar de modulação. Observa-se que os algoritmos FF-Dis e LMCI apresentam margens próximas dos valores apresentados na Seção 4.1, acima de 2 dB. Para o algoritmo SAIR a margem adotada é muito próxima do limite avaliado 1,5 $\mathrm{dB}$, o que pode prejudicar o estabelecimento de futuros circuitos na rede. Por outro lado para o algoritmo RQoTO a margem adotada é muito alta, cerca de 4 a $4,5 \mathrm{~dB}$, o que provoca a subutilização do recursos da rede. Isso se deve ao fato do algoritmo escolher os candidatos com maior margem $(>\Delta S N R)$ dentre as rotas e modulações avaliadas. De acordo com os resultados apresentados na Seção 4.1, as melhores margens adotadas para o estabelecimento dos circuitos esta próximo de $2 \mathrm{~dB}$. Basicamente uma margem inferior a este valor prejudica o estabelecimento de futuros circuitos, enquanto uma margem muito superior a este valor subutiliza o canal óptico. Os resultados apresentados mostram que o algoritmo LMCI esta de acordo com o limites previamente avaliados.

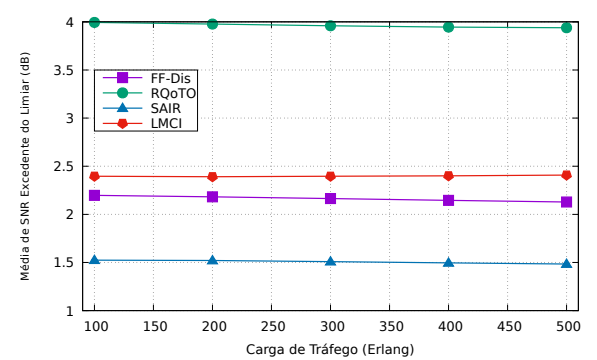

(a) Topologia USANet

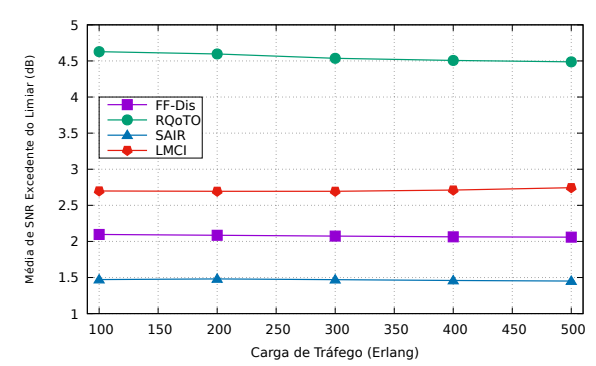

(b) Topologia PanEuro

Figura 6. Percentual Médio de SNR Excedente do Limiar (\%).

\subsection{Taxa de Bloqueio de Chamada}

A taxa de bloqueio de chamada (Figura 7) reflete a quantidade de requisições bloqueadas na rede, quanto maior essa taxa, maior é o número de demandas bloqueadas. Pode-se observar que o algoritmo LMCI obteve o menor bloqueio de chamada frente aos demais algoritmos comparados, apresentando um ganho de até $98 \%$ para o algoritmo RQoTO e em média 33\% melhor que os algoritmos FF-Dis e SAIR para ambas as topologias. Isso se deve ao fato do LMCI explorar melhor o uso do formatos de modulação adotados nos circuitos, estabelecendo uma margem de segurança suficiente para garantir uma eventual degradação do canal. Além disso, o fato de escolher modulações mais robustas sem alterar a quantidades de espectro alocado (Figura 4, etapa IV) beneficia o fortalecimento do circuito adotando margens ainda maiores sem prejuízos no desempenho de espectro.

\subsection{Tempo Médio de Atendimento da Demanda (ms)}

Para investigar o desempenho de tempo de execução dos algoritmos avaliados, foram calculados o tempo médio que o algoritmo RMLSA leva para atender ou bloquear uma demanda. Diversos testes foram realizados em uma máquina com processador de 2,40 GHz (Intel(R) Xeon(R) CPU E7-4870) e 16 GB de RAM. A Figura 8 mostra o tempo médio de decisão da engenharia de tráfego EON sob várias cargas de tráfego em ambas as topologias. Observa-se que os algoritmos RQoTO e SAIR levam bastante tempo para atender uma demanda na rede, entre 10 até $70 \mathrm{~ms}$. Isso de deve ao fato destes algoritmos avaliarem vários candidatos antes de tomar a decisão de alocação do circuito. Por 


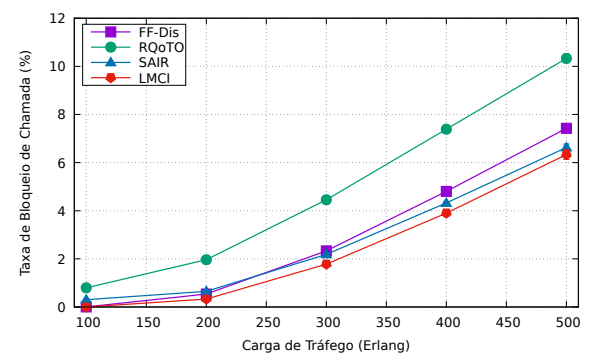

(a) Topologia USANet

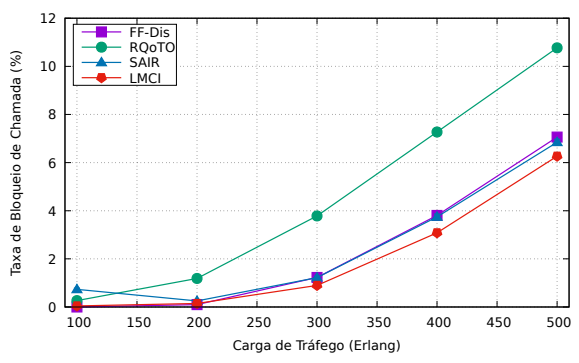

(b) Topologia PanEuro

Figura 7. Taxa de Bloqueio de Chamadas.

outro lado o algoritmo LMCI obtém um desempenho próximo ao algoritmo FF-Dis que considera apenas o limiar de distância na escolha dos candidatos para o estabelecimento do circuito, em torno de $2 \mathrm{~ms}$ em média. Isto acontece, pois o algoritmo LMCI evita percorrer todas as modulações da rede devido a sua tonificação de circuito que adianta a avaliação do laço das modulações consideradas (vide Figura 4).

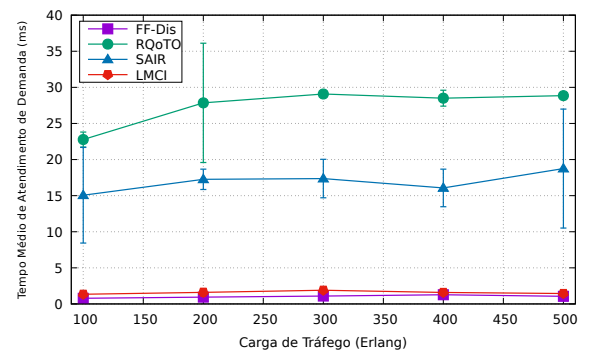

(a) Topologia USANet

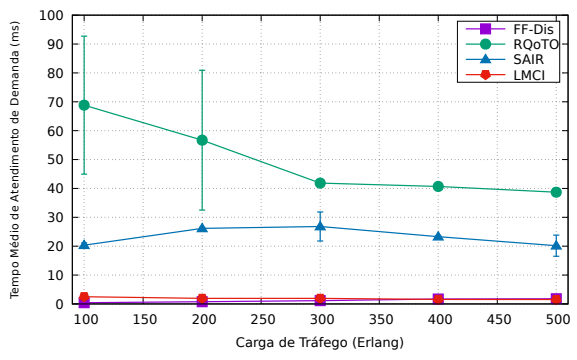

(b) Topologia PanEuro

Figura 8. Tempo de processamento da Simulação.

\section{Conclusão}

Este trabalho estudou as margens de QoT adotadas na alocação de circuitos ópticos em algoritmos RMLSA. Foram avaliados os algoritmos tradicionais da literatura que quantificam o QoT por meio do alcance máximo transparente da modulação em diversos cenários. O estudo mostrou que em muitos casos, a literatura subutiliza o canal óptico, utilizando margens de QoT superdimensionadas. O trabalho mostrou como explorar eficientemente estes fatores para contribuir para a elaboração de técnicas mais eficientes no desenvolvimento de soluções de engenharia de tráfego das redes de núcleo. Para demonstrar a eficiência de explorar melhor as margens de QoT adotadas em algoritmos RMLSA, este artigo propôs um novo algoritmo RMLSA que categoriza as margens mínimas que devem ser adotadas para o estabelecimento de novos circuitos, tendo como base a sua degeneração esperada e o impacto que ele causará aos circuitos ópticos já estabelecidos. Os resultados de simulação mostraram que o esquema de margem dinâmico proposto associada ao fortalecimento dos circuitos óticos apresentam melhores resultados que algoritmos da literatura que propõem uma margem mais justa ou que avaliam o QoT por meio do alcance máximo transparente. A proposta mostrou uma redução em média de $33 \% \mathrm{em}$ termos de bloqueio de chamadas com um desempenho de tempo de execução semelhante a algoritmos tradicionais que avaliam o QoT pela distância. 


\section{Referências}

Beyranvand, H. and Salehi, J. A. (2013). A quality-of-transmission aware dynamic routing and spectrum assignment scheme for future elastic optical networks. Journal of Lightwave Technology, 31(18):3043-3054.

Brasileiro, I., Valdemir, J., and Soares, A. (2019). Regenerator assignment with circuit invigorating. Optical Switching and Networking, 34:58 - 66.

Chatterjee, B., Sarma, N., and Oki, E. (2015). Routing and spectrum allocation in elastic optical networks: A tutorial. IEEE Communications Surveys Tutorials, 17(3):1776-1800.

Chen, X., Li, J., Zhu, P., Tang, R., Chen, Z., and He, Y. (2015). Fragmentation-aware routing and spectrum allocation scheme based on distribution of traffic bandwidth in elastic optical networks. Journal of Optical Communications and Networking, 7(11):1064-1074.

Christodoulopoulos, K., Tomkos, I., and Varvarigos, E. (2011). Elastic bandwidth allocation in flexible OFDM-based optical networks. Journal of Lightwave Technology, 29(9):1354-1366.

Costa, L. R. and Drummond, A. C. (2017). New distance-adaptive modulation scheme for elastic optical networks. IEEE Communications Letters, 21(2):282-285.

Costa, L. R. and Drummond, A. C. (2020). ONS - optical network simulator. https: //gitlab.com/get-unb/ons.

Feng, M., Jiang, R., and Shen, J. (2017). A segmentation modulation based spectral allocation scheme for elastic optical networks. In 2017 16th International Conference on Optical Communications and Networks (ICOCN), pages 1-3.

Fontinele, A., Santos, I., Jr, J. L., Soares, A., Monteiro, J. S., and Campelo, D. R. (2018). Alocação de Espectro com Redução de Interferências entre Circuitos em Redes ópticas Elásticas. In SBRC 2018, Campos do Jordão, São Paulo.

Fontinele, A., Santos, I., Neto, J. N., Campelo, D. R., and Soares, A. (2017). An Efficient IARMLSA Algorithm for Transparent Elastic Optical Networks. Computer Networks, 118:1-14.

Guo, P., Hou, W., Wu, J., and Sun, Z. (2015). Modulation adaptive grooming in green elastic optical networks. In 14th International Conference on Optical Communications and Networks (ICOCN), 2015, pages 1-3.

Johannisson, P. and Agrell, E. (2014). Modeling of nonlinear signal distortion in fiber-optic networks. Journal of Lightwave Technology, 32(23):3942-3950.

Pointurier, Y. (2017). Design of low-margin optical networks. IEEE/OSA Journal of Optical Communications and Networking, 9(1):A9-A17.

Walkowiak, K., Klinkowski, M., and Lechowicz, P. (2017). Influence of modulation format transmission reach on performance of elastic optical networks. In 2017 19th International Conference on Transparent Optical Networks (ICTON), pages 1-4.

Wan, X., Hua, N., and Zheng, X. (2012). Dynamic routing and spectrum assignment in spectrumflexible transparent optical networks. IEEE/OSA Journal of Optical Communications and Networking, 4(8):603-613. 